\title{
Serum homocysteine is associated with polycystic ovarian syndrome in Jordan
}

\author{
NESREEN SAADEH ${ }^{1}$, MAHMOUD A. ALFAQIH $^{2}$, HANEEN MANSOUR $^{2}$, \\ YOUSEF S. KHADER ${ }^{3}$, RAMI SAADEH ${ }^{3}$, AHMED AL-DWAIRI $^{2}$ and MOHAMAD NUSIER ${ }^{2}$
}

Departments of ${ }^{1}$ Internal Medicine, ${ }^{2}$ Physiology and Biochemistry, and ${ }^{3}$ Family Medicine and Public Health, School of Medicine, Jordan University of Science and Technology, Irbid 22110, Jordan

Received March 26, 2018; Accepted August 28, 2018

DOI: $10.3892 /$ br.2018.1149

\begin{abstract}
Polycystic ovarian syndrome (PCOS) is a prevalent endocrinopathy among women of a reproductive age. Although not included in the diagnostic criteria, insulin resistance (IR) is a major characteristic of PCOS and may contribute to its development. The exact cause of IR remains unknown but appears to be multifactorial. Changes in the levels of leptin, adiponectin, branched-chain amino acids (BCAAs) and/or homocysteine have been reported in women with PCOS. However, the relative contribution of the aforementioned metabolites to PCOS has not been tested in Jordan. In the present study, 154 women diagnosed with PCOS and 151 normally menstruating women matched by age and body mass index (BMI) were recruited. The levels of leptin, adiponectin, BCAAs, homocysteine and 5-methyltetrahydrofolate (5-MTHF) were measured in the serum of the recruited participants. It was revealed that homocysteine levels were significantly elevated in women with PCOS compared with normally menstruating women $(\mathrm{P}<0.0001)$, while 5-MTHF $(\mathrm{P}=0.024)$, leptin $(\mathrm{P}=0.027)$ and adiponectin $(\mathrm{P}=0.010)$ levels were significantly lower. In multivariate analysis, serum homocysteine had the strongest association with PCOS and significantly increased its risk $[\mathrm{P}<0.0001$; odds ratio 1.217; $95 \%$ confidence interval (CI) 1.157-1.280]. With an area under
\end{abstract}

Correspondence to: Dr Mahmoud A. Alfaqih, Department of Physiology and Biochemistry, School of Medicine, Jordan University of Science and Technology, M3L0, Irbid 22110, Jordan

E-mail: maalfaqih@just.edu.jo

Abbreviations: PCOS, polycystic ovarian syndrome; IR, insulin resistance; BCAAs, branched-chain amino acids; 5-MTHF, 5-methyltetrahydrofolate; MTHFR, methylenetetrahydrofolate reductase; KAUH, King Abdullah University Hospital; ELISA, enzyme-linked immunosorbent assay; PCR, polymerase chain reaction; RFLP, restriction fragment length polymorphism; ROC, receiver operating characteristic

Key words: polycystic ovarian syndrome, insulin resistance, homocysteine, 5-methyltetrahydrofolate, methylenetetrahydrofolate reductase the curve of 0.855 (95\% CI 0.811-0.898) in receiver operating characteristic analysis, serum homocysteine was determined to be a good predictor for PCOS diagnosis based on Rotterdam guidelines. It was concluded that serum levels of homocysteine are elevated in women with PCOS in Jordan independent of age, BMI, or leptin, adiponectin and BCAAs levels.

\section{Introduction}

Polycystic ovarian syndrome (PCOS) is an endocrine disorder that occurs in women of reproductive age (1). According to the Rotterdam guidelines, diagnosis with PCOS requires the presence of at least two of the following; irregular periods, increased androgen secretion and/or polycystic ovaries (2). Although not included in the diagnostic criteria, the majority of women with PCOS are insulin resistant (3). Insulin resistance (IR) in women with PCOS increases their risk of type 2 diabetes mellitus and other co-morbidities (3). Furthermore, IR in these women appears to contribute to PCOS development (4). Indeed, weight loss (known to increase insulin sensitivity) is associated with a reduction in PCOS co-morbidities or even disease resolution (5).

In addition to the dysregulation of insulin levels/activity, IR is associated with altered levels of a number of other hormones including adiponectin (6), leptin (7) and irisin (8). It is therefore unsurprising that IR causes dysregularities in carbohydrate, fat and protein metabolism. For example, homocysteine and the branched-chain amino acids (BCAAs) (isoleucine, leucine and valine) are often elevated in the serum of individuals with increased IR $(9,10)$. The function of homocysteine in IR has received attention recently as it is atherogenic and prothrombotic and may contribute to the increased prevalence of cardiovascular complications in individuals with IR (11).

Homocysteine is synthesized from methionine (12). Once formed, homocysteine may be methylated back into methionine through a reaction that requires 5-methyltetrahydrofolate (5-MTHF) and the methylcobalamin form of vitamin $\mathrm{B}_{12}(12)$. Alternatively, through a transsulfuration reaction, the sulfur group of homocysteine may be transferred to serine to form cysteine and $\alpha$-ketobutyrate (12). Transsulfuration of homocysteine requires the activity of two enzymes; cystathionine synthase and cystathionine lyase (12). The above two enzymes use a pyridoxal-phosphate (i.e., vitamin $\mathrm{B}_{6}$ ) as a cofactor (13). 
Increased insulin levels, a feature of IR, are believed to raise homocysteine levels by inhibiting the activity of the hepatic cystathionine synthase (14). However, the exact mechanism underlying the association between IR and higher homocysteine levels is yet to be completely understood.

Homocysteine levels in the blood may additionally be influenced by the activity of the enzyme methylenetetrahydrofolate reductase (MTHFR), the rate-limiting enzyme of the methyl cycle (15). MTHFR catalyzes the conversion of 5,10-methylenetetrahydrofolate to 5-MTHF, a co-substrate of the reaction that converts homocysteine to methionine (15). This explains why individuals that have MTHFR variants with low activity levels often have higher homocysteine levels (15). One of the most commonly investigated variants of MTHFR is caused by a single nucleotide polymorphism in the coding region 'C677T' (16). Higher homocysteine levels caused by this variant may thus increase the risk of PCOS.

Given the association between IR and PCOS and the association between hyperinsulinemia and increased levels of blood homocysteine, previous studies have investigated the association between PCOS and homocysteine with conflicting results (17-19). In the present study, the association between homocysteine, the C677T MTHFR variant was assessed in individuals with PCOS in a Jordanian population. The strength of the association of homocysteine with PCOS relative to other metabolites known to be involved with IR (leptin, adiponectin and BCAAs) were additionally assessed.

\section{Materials and methods}

Study design and patients. This was a prospective frequency matched case-control study. The study population was previously used to establish an association between lower levels of serum adiponectin and PCOS (20). The project was ethically approved by the appropriate Institutional Review Boards (290/2016) affiliated with the Jordan University of Science and Technology (Irbid, Jordan). Patients provided written informed consent required for participation in the present study.

Patients with PCOS were recruited from the Gynecology clinic of the King Abdullah University Hospital (KAUH) between September 2016 and March 2017. KAUH is a tertiary hospital affiliated with Jordan University of Science and Technology.

Normally menstruating women were recruited from other clinics within KAUH provided they met the eligibility criteria described below. To participate in the study, women with PCOS had to have been diagnosed with PCOS by at least two gynecologists from KAUH according to the Rotterdam guidelines (21). Normally menstruating women had a regular menstrual cycle in terms of duration and quantity for at least six months prior to enrollment, and had no clinical or biochemical signs of hyperandrogenism. Additionally, their ovaries were free of follicles upon a mandatory ultrasound examination performed by the study gynecologist. Women who were affected with any of the following: Cushing's syndrome, syndromes of severe IR, androgen secreting neoplasms, thyroid dysfunction, congenital adrenal hyperplasia or hyperprolactinemia were excluded from the study. The height, weight and age of the patients were recorded at the time of the patient visit and interview. The following formula: body mass index $(\mathrm{BMI})=$ weight $(\mathrm{kg}) /[\text { height }(\mathrm{m})]^{2}$ was used for BMI calculation.

A total of 154 women with PCOS (mean age, 23.9 \pm 5.1 years old) and 151 normally menstruating women (mean age, $24.2 \pm 5.1$ years old) agreed to participate in the study. The age range of the subjects was between 14 and 34 years. The study aimed to detect a difference of 5 units (equivalent to a small effect size) in homocysteine between the two groups assuming that the common standard deviation is 11 and the level of significance $(\mathrm{P}<0.05)$ exceeded $80 \%$ (power $=93 \%)$. The power analysis was calculated using $G^{*}$ Power software version 3.1.9 (Department of Psychology, University of Düsseldorf, Düsseldorf, Germany) (22) and Epicalc 2000 software version 1.5 (Prince of Songkla University, Hat Yai, Thailand) (23).

Collection of blood and serum samples. Blood withdrawal was performed subsequent to an overnight fast of $12 \mathrm{~h}$. Blood samples were distributed into an EDTA tube (Al-Hanoof Factory, Amman, Jordan) and a plain tube with gel clot activator (Al-Hanoof Factory). Blood in the EDTA tubes was directly used for DNA extraction while blood in the plain tubes was used for the recovery of serum following centrifugation at $4,000 \mathrm{x} \mathrm{g}$ for $7 \mathrm{~min}$ at room temperature. The recovered serum was stored at $-80^{\circ} \mathrm{C}$ until it was further used to determine the levels of homocysteine, 5-MTHF, leptin, adiponectin and BCAAs.

Biochemical measurements. Serum homocysteine, 5-MTHF, leptin and adiponectin levels were measured using enzyme-linked immunosorbent assay (ELISA) kits according to the manufacturer's protocol. The homocysteine ELISA kit was purchased from Cell Biolabs, Inc. (cat no. STA-670; San Diego, CA, USA), the 5-MTHF ELISA kit was purchased from Cusabio Technology LLC (cat no. CSB-E17109h; Houston, TX, USA) while the leptin (cat no. DY398) and adiponectin (cat no. DY1065) ELISA kits were purchased from R\&D Systems, Inc. (Minneapolis, MN, USA). To measure homocysteine levels, serum samples were diluted 10-fold. Serum samples were diluted 100-fold to measure the 5-MTHF and leptin levels, and were diluted 8,000-fold to measure adiponectin levels. All dilutions were prepared in phosphate buffer saline containing $0.1 \%$ bovine serum albumin (cat no. P3688; Sigma-Aldrich; Merck KGaA, Darmstadt, Germany).

On the other hand, serum BCAAs levels were measured using a commercially available kit purchased from Sigma-Aldrich (Merck KGaA) with leucine used as the standard. The assay used a coupled enzymatic reaction to determine the concentration of all three BCAAs, resulting in a colorimetric product. For all ELISAs performed, the absorbance was measured using an ELx800 microplate reader (BioTek Instruments, Inc., Winooski, VT, USA). A wavelength of $450 \mathrm{~nm}$ was used for the measurements.

DNA extraction and genotyping. Genomic DNA was purified from blood samples in EDTA tubes using a QIAamp DNA Blood Mini kit purchased from Qiagen GmbH (Hilden, Germany) according to the manufacturer's protocol. Following DNA extraction, the final DNA concentration was measured spectrophotometrically using an ND-2000 Nanodrop (Thermo 
Fisher Scientific, Inc., Waltham, MA, USA). Polymerase chain reaction-restriction fragment length polymorphism (PCR-RFLP) was used to genotype the C677T variant of the MTHFR gene. The final volume of the PCR reaction was $25 \mu \mathrm{l}$. The reaction mixture contained GoTaq ${ }^{\circledR}$ Green Master Mix (Promega Corporation, Madison, WI, USA), 5 ng DNA and $0.4 \mu \mathrm{M}$ primers (forward and reverse). The sequence of the C677T variant of the MTHFR gene forward primer was 5'-GTCGGTGCATGCCTTCAC'-3 while the sequence of the reverse primer was 5'-AGCATATCAGTCATGAGCCC'-3. The PCR was run under the following thermocycling conditions: Initial denaturation at $95^{\circ} \mathrm{C}$ for $3 \mathrm{~min}$, followed by 34 cycles of denaturation at $95^{\circ} \mathrm{C}$ for $3 \mathrm{~min}$, annealing at $65^{\circ} \mathrm{C}$ for $30 \mathrm{sec}$ and extension at $72^{\circ} \mathrm{C}$ for one min and a final extension at $72^{\circ} \mathrm{C}$ for $5 \mathrm{~min}$. The above PCR reaction produced an amplicon $333 \mathrm{bps}$ in length. In order to genotype the patients for the different genotype categories of C677T, the above PCR amplicon was digested with TaqaI (New England BioLabs, Inc., Ipswitch, MA, USA) at $65^{\circ} \mathrm{C}$ for $1 \mathrm{~h}$. The undigested PCR amplicons and the products following restriction enzyme digestion were electrophoresed on $3 \%$ agarose gel containing ethidium bromide (cat no. 1613024; Bio-Rad Laboratories, Inc., Hercules, CA, USA). Ultraviolet light was used to visualize the products. Direct Sanger sequencing was performed at the Princess Haya Biotechnology Center (KAUH, Irbid, Jordan) to confirm the genotyping results.

Statistical analysis. The Statistical Package for Social Studies (SPSS) software version 22 (SPSS, Inc., Chicago, IL, USA) was used to conduct all statistical analyses. The data were presented as the mean \pm standard deviation. An unpaired Student's t-test was used to examine if a statistically significant difference was present in serum homocysteine, 5-MTHF, leptin, adiponectin, BCAAs, age or BMI between normally menstruating and PCOS women. A Pearson's $\chi^{2}$ was used to examine if an association existed between the different genotype categories of C677T with PCOS. To assess if significant differences existed in homocysteine or 5-MTHF serum levels between the different genotype classes of MTHFR C677T (CC, CT or TT), one-way analysis of variance followed by a post hoc Tukey's test was used.

To assess the strength of the association of homocysteine with PCOS, multivariate logistic regression analysis was used with the following variables in the model: Age, BMI, homocysteine, 5-MTHF, leptin, adiponectin, BCAAs and the C677T different genotypes. A receiver operating curve (ROC) analysis was used to evaluate the performance of homocysteine as a diagnostic test for PCOS. $\mathrm{P}<0.05$ was considered to indicate a statistically significant difference.

\section{Results}

Patient characteristics and biochemical profiles. A total of 154 PCOS and 151 normally menstruating women consented to participate in the present study. Their ages ranged between 14 and 34 years. Fasting serum homocysteine levels were significantly higher in patients with PCOS compared with normally menstruating women $(\mathrm{P}<0.0001$; Table I). On the other hand, women with PCOS had significantly lower levels of fasting leptin $(\mathrm{P}=0.027)$, adiponectin $(\mathrm{P}=0.010)$ and 5-MTHF
Table I. Baseline characteristics of the study participants.

\begin{tabular}{lccc}
\hline & $\begin{array}{c}\text { Normal } \\
\text { menstruating } \\
\text { patients }\end{array}$ & $\begin{array}{c}\text { Patients } \\
\text { with PCOS }\end{array}$ & P-value \\
\hline Age, years & $24.35 \pm 5.10$ & $23.85 \pm 5.12$ & 0.3971 \\
BMI, kg/m ${ }^{2}$ & $26.23 \pm 5.82$ & $26.57 \pm 5.98$ & 0.5812 \\
BCAAs, $\mu$ mol/1 & $373.68 \pm 78.72$ & $385.23 \pm 89.93$ & 0.2423 \\
Leptin, ng/ml & $27.72 \pm 22.10$ & $22.77 \pm 16.15$ & 0.0270 \\
Adiponectin, & $7.87 \pm 4.79$ & $6.61 \pm 3.54$ & 0.0103 \\
$\mu$ g/dl & & & \\
5-MTHF, ng/ml & $13.79 \pm 4.88$ & $12.61 \pm 3.96$ & 0.0243 \\
Homocysteine, & $14.23 \pm 5.53$ & $29.03 \pm 11.80$ & $<0.0001$ \\
$\mu$ mol/l & & & \\
\hline
\end{tabular}

Data are presented as the mean \pm standard deviation. P-values were calculated using an unpaired Student's t-test. PCOS, polycystic ovarian syndrome; BMI, body mass index; BCAAs, branched chain amino acids; 5-MTHF, 5-methyltetrahydrofolate.

Table II. Multivariate analysis with age, BMI, BCAA, leptin, adiponectin, 5-MTHF and homocysteine as variables in the model.

\begin{tabular}{lccr}
\hline Variable & OR & 95\% CI & P-value \\
\hline Age, years & 0.961 & $0.897-1.029$ & 0.2531 \\
BMI, kg/m ${ }^{2}$ & 1.072 & $0.993-1.157$ & 0.0763 \\
BCAA, $\mu \mathrm{mol} / \mathrm{l}$ & 1.000 & $0.995-1.004$ & 0.8742 \\
Leptin, ng/ml & 0.960 & $0.934-0.986$ & 0.0031 \\
Adiponectin, $\mu \mathrm{g} / \mathrm{ml}$ & 0.984 & $0.899-1.076$ & 0.7203 \\
5-MTHF, ng/ml & 0.954 & $0.884-1.031$ & 0.2343 \\
Homocysteine, $\mu \mathrm{mol} / \mathrm{l}$ & 1.217 & $1.157-1.280$ & $<0.0001$ \\
\hline
\end{tabular}

P-values were calculated by multivariate logistic regression. OR, odds ratio; BMI, body mass index; BCAA, branched chain amino acids; 5-MTHF, 5-methyltetrahydrofolate; CI, confidence interval.

$(\mathrm{P}=0.024$; Table I) compared with normally menstruating women.

Associations between homocysteine and PCOS. The present study aimed to assess whether the serum homocysteine levels remained significantly associated with PCOS subsequent to adjusting for other co-variables. To achieve this, multivariate logistic regression analysis was used and it was revealed that serum homocysteine increased the risk of and demonstrated the strongest association with PCOS $[\mathrm{P}<0.0001$; odds ratio, 1.217; 95\% confidence interval (CI), 1.157-1.280; Table II].

Correlation between homocysteine and 5-MTHF. Serum homocysteine levels were expected to be negatively correlated with 5-MTHF levels. In order to assess this association, correlation analysis was performed to determine any correlation between homocysteine and the other variables in the 
Table III. Correlation between homocysteine and other variables.

\begin{tabular}{lcccccc}
\hline Variable & Age, years & BMI, $\mathrm{kg} / \mathrm{m}^{2}$ & BCAAs, $\mu \mathrm{mol} / \mathrm{l}$ & Leptin, $\mathrm{ng} / \mathrm{ml}$ & Adiponectin, $\mu \mathrm{g} / \mathrm{ml}$ & 5-MTHF, $\mathrm{ng} / \mathrm{m}$ \\
\hline Homocysteine, $\mu \mathrm{mol} / \mathrm{l}$ & & & & & & \\
$\quad$ r-value & -0.037 & 0.064 & 0.149 & 0.005 & -0.172 & -0.168 \\
P-value & 0.530 & 0.283 & 0.012 & 0.934 & 0.004 & 0.005
\end{tabular}

P-values were calculated using a two-tailed Student's t-test. BMI, body mass index; BCAA, branched chain amino acids; 5-MTHF, 5-methyltetrahydrofolate.

population (including age, BMI, BCAA, leptin, adiponectin and 5-MTHF levels). The results revealed that a significant negative correlation existed between homocysteine and 5-MTHF ( $\mathrm{r}=-0.168, \mathrm{P}=0.005)$, and between homocysteine and adiponectin $(\mathrm{r}=-0.172, \mathrm{P}=0.004)$. On the other hand, a positive significant correlation existed between homocysteine and BCAAs ( $\mathrm{r}=0.149, \mathrm{P}=0.012$; Table III).

Effect of the MTHFR C677T polymorphism on the serum levels of homocysteine and 5-MTHF. 5-MTHF levels may be affected by the activity of MTHFR. To assess whether the C677T MTHFR variant that may affect the activity of MTHF is associated with PCOS and/or affects 5-MTHF and homocysteine levels, a PCR-RFLP strategy was developed to genotype the study participants for the C677T MTHFR variant (Fig. 1A). The results revealed that the TT genotype of C677T was associated with significantly lower levels of serum 5-MTHF compared with the CC genotype ( $\mathrm{P}=0.0186$; Fig. 1B). Furthermore, the TT genotype of C677T was associated with significantly higher levels of serum homocysteine compared with the $\mathrm{CT}$ genotype $(\mathrm{P}=0.0369)$ or the $\mathrm{CC}$ genotype $(\mathrm{P}=0.007$; Fig. 1C). No significant association existed between any of the C677T genotypes and PCOS ( $\mathrm{P}=0.505$; Table IV).

Evaluation of the accuracy of using serum homocysteine in PCOS diagnosis. Given the results of the present study, the accuracy of serum homocysteine as a predictor for PCOS was further examined. To achieve this, ROC analysis was performed (Fig. 2). With an area under the curve of 0.855 (95\% CI, 0.811-0.898) and a cut-off level of $20.05 \mu \mathrm{mol} / 1$, the results revealed that serum homocysteine levels are a good predictor for PCOS if diagnosis is based on the Rotterdam guidelines. The analysis revealed that at the cut-off value, serum homocysteine has a sensitivity of $73.65 \%$ and a specificity of $86.33 \%$ in predicting PCOS.

\section{Discussion}

The results of the present study provide a tentative link between homocysteine metabolism and PCOS. If such a link is validated in larger studies, this will underscore approaches that may be utilized to better manage the disease and/or its associated co-morbidities. The most notable result was that the serum levels of homocysteine were dramatically elevated in women with PCOS. Importantly, it was observed that higher homocysteine levels remain associated with PCOS even subsequent to adjusting for other potentially confounding variables
A
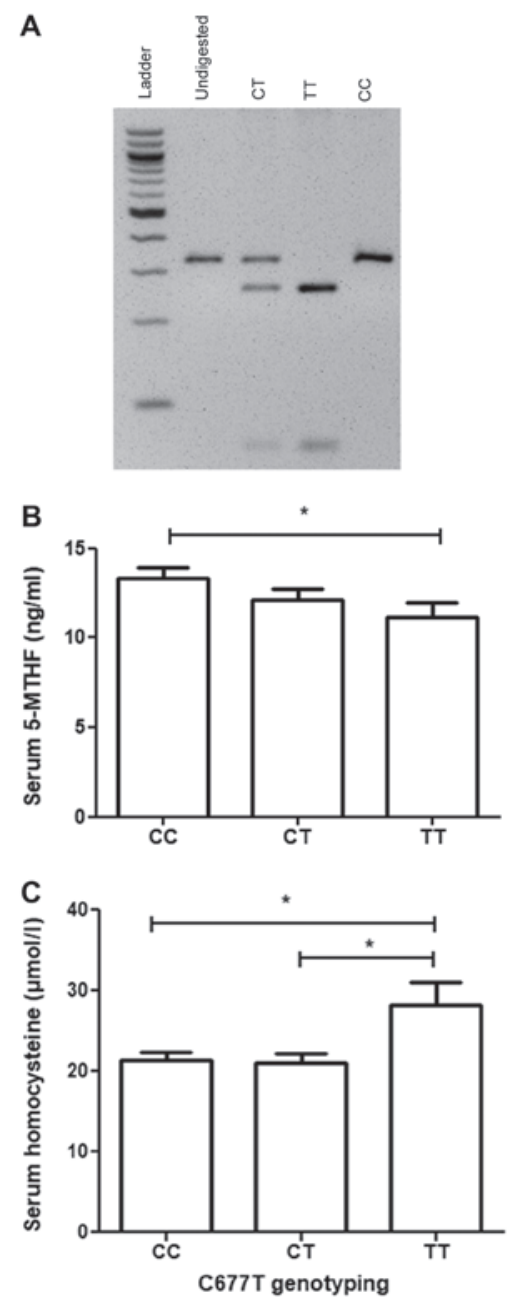

Figure 1. Effect of the genotype class of the C667T polymorphism of MTHFR on serum homocysteine and 5-MTHF levels. (A) A 2\% agarose gel image of the different genotype classes of the C677T polymorphism of MTHFR observed in the patients following the use of a polymerase chain reaction-restriction fragment length polymorphism strategy. Patients were then categorized according to their C677T genotype class and analysis of variance followed by a post-hoc Tukey's test was then performed to determine if significant differences existed in (B) serum 5-MTHF or (C) serum homocysteine levels between the different genotype classes. The concentration of serum homocysteine is expressed in $\mu \mathrm{mol} / 1$ while the serum concentration of 5-MTHF is expressed in $\mathrm{ng} / \mathrm{ml}$. The error bars represent the standard error of the mean. ${ }^{*} \mathrm{P}<0.05$ with comparisons shown by lines. MTHFR, methylenetetrahydrofolate reductase; 5-MTHF, 5-methyltetrahydrofolate.

(including age, BMI, BCAAs, adiponectin and leptin) previously reported to be associated with PCOS. Furthermore, it was revealed that homocysteine levels were negatively 
Table IV. Genotype frequencies of rs1801133 in normal menstruating women and women with PCOS.

\begin{tabular}{lccc}
\hline Genotype & $\begin{array}{c}\text { Normal } \\
\text { menstruating } \\
(\mathrm{n}=149)\end{array}$ & $\begin{array}{c}\text { PCOS } \\
(\mathrm{n}=154)\end{array}$ & P-value \\
\hline CC & $70(46.4 \%)$ & $77(50.0 \%)$ & 0.505 \\
CT & $69(45.7 \%)$ & $61(39.6 \%)$ & \\
TT & $12(7.9 \%)$ & $16(10.4 \%)$ & \\
\hline
\end{tabular}

Data are presented as percentages. P-values were calculated by Pearson $\chi^{2}$ test. PCOS, polycystic ovarian syndrome.

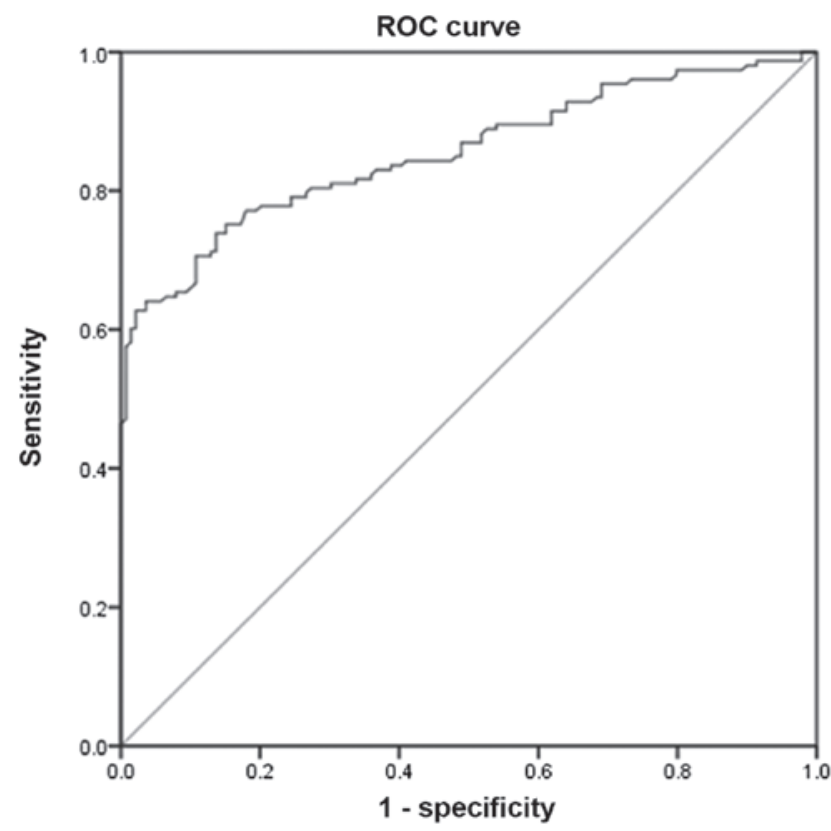

Figure 2. ROC analysis of serum homocysteine as a diagnostic marker of polycystic ovarian syndrome. ROC, receiver operating characteristics.

correlated with 5-MTHF levels and were affected by the C677T polymorphism of the MTHFR enzyme; a polymorphism known to affect MTHFR activity and therefore affect 5-MTHF levels (24).

Although IR is not required to establish PCOS diagnosis, it is a common feature of PCOS and appears to be involved in the pathogenesis of this disorder (25). Indeed, women with PCOS benefit from weight reduction and other lifestyle changes that increase insulin sensitivity (25). The precise cause behind IR in PCOS appears to be multifactorial. Dysregulation in the levels of a number of biochemical markers (including leptin, adiponectin and BCAAs) was proposed to serve a role in establishing and/or maintaining the PCOS phenotype with conflicting evidence which either supports or refutes the association (25). The strength of the association of these biochemical markers with PCOS was not adequately assessed previously. The present study revealed that relative to leptin, adiponectin and BCAAs, homocysteine is the most strongly associated with PCOS. The importance of this observation for PCOS diagnosis and treatment requires further investiga- tion. For example, it remains to be determined whether the elevation that was observed in homocysteine levels in women with PCOS is involved in the disease pathogenesis or is simply an outcome of this disorder. The answer to this question requires experiments in PCOS animal models, where the effect of altering the levels of homocysteine on PCOS may be investigated in vivo (26). If higher homocysteine levels are confirmed to be directly or indirectly involved in PCOS development, this will encourage therapeutic approaches to mitigate PCOS through lowering serum homocysteine levels. Indeed, the effective lowering of homocysteine levels may be achieved through the administration of 5-MTHF, methylcobalamin (vitamin $\mathrm{B}_{12}$ ) and/or pyridoxal phosphate (vitamin $\mathrm{B}_{6}$ ) combinations (27). This approach may have utility in women with PCOS, as the present study revealed that homocysteine levels are negatively correlated with 5-MTHF levels indicating that the administration of 5-MTHF may lower homocysteine levels and potentially affect PCOS and/or its co-morbidities; this requires formal testing in well-designed clinical trials.

Metabolic dysfunction observed in women with PCOS is associated with an increase in cardiovascular disease risk markers (28). Whether this increase reflects a higher prevalence of cardiovascular morbidity and mortality among women with PCOS remains a matter of debate (28). A number of studies have reported an association between serum homocysteine levels and an increased prevalence of cardiovascular morbidity and mortality (29-31). Given this association, it is plausible that women with higher serum homocysteine levels may have a higher prevalence of cardiovascular-associated morbidity and mortality, particularly women that have inactivating base substitutions in the MTHFR gene. This association, however, requires formal testing and is an ongoing effort.

Ethnic differences exist in the clinical and biochemical presentation of PCOS. For example, Asian women that have PCOS are often shorter in height, have a lower BMI and a less severe hyperandrogenic phenotype (32). On the contrary, women with PCOS of Hispanic origin are usually more likely to be obese and have a predilection to type 2 diabetes mellitus and the metabolic syndrome (33). In the present investigation, it was revealed that women with PCOS of Middle Eastern origin have dramatically elevated levels of serum homocysteine. Further investigations are required to test if this elevation is common among women with PCOS of different ethnicities. Of note, a previous meta-analysis of 34 studies, which included 1,718 women with PCOS and 1,399 controls from populations of various ethnicities and genetic backgrounds, revealed that serum homocysteine levels are elevated in women with PCOS even subsequent to adjustment for obesity, IR and androgen levels (34). The results of the present study combined with the results of the aforementioned study provide strong support to the argument that ethnic variation may not influence the association between PCOS and elevated serum homocysteine levels.

PCOS is a disorder of heterogeneous clinical and biochemical presentation. Consensus on diagnostic criteria for PCOS was only reached in 2003 and was mainly based on expert opinions (21). Furthermore, ethnic differences exist in disease presentation (35). All of these factors result in the diagnosis of PCOS being a challenging task and there are a number of reports where women with PCOS 
have expressed their dissatisfaction with the time it took to reach diagnosis and with the lack of adequate education on the disease $(36,37)$. Diagnosis of PCOS is particularly challenging among adolescents for the following reasons; i) acne not associated with PCOS is common among this age group; and ii) irregularities in the menstrual cycle are prevalent in the first years that follow menarche (38). A number of experts have suggested continuous refinement of the biochemical and clinical criteria required to establish PCOS diagnosis (39). In the present investigation, ROC analysis revealed that serum homocysteine is a good biomarker for diagnosis. However, this result requires larger studies among different ethnicities and different age groups to validate the potential use of serum homocysteine as a diagnostic biomarker for PCOS, at least in Jordan. Interestingly, however, given the meta-analysis performed by Meng et al (34) and described above which revealed that serum homocysteine levels were elevated in women with PCOS of various ethnicities, serum homocysteine may prove to be a universal diagnostic biomarker of PCOS.

The TT genotype of the C677T polymorphism of MTHFR was associated with higher levels of serum homocysteine in the present study and serum homocysteine was associated with PCOS; however, no association was detected between the aforementioned polymorphism of MTHFR with PCOS. This may be explained by the sample size of patients used in this study, which may not have been large enough to detect such an association, particularly as the total number of individuals that carried the TT genotype in the present study was only 28 . Additionally, the presence of other polymorphisms associated with the C677T polymorphism may have cancelled the effect of the C677T polymorphism on PCOS risk.

Although not the primary goal of the present study, it was revealed that serum leptin levels were lower in women with PCOS compared with normally menstruating women. This association remained significant even subsequent to adjusting for age, BMI, leptin, adiponectin and BCAAs in multivariate analysis. Leptin is a hormone secreted from the adipocytes (40). Leptin and its receptor serve an important role in regulating appetite, food intake and energy expenditure through its effect on the hypothalamic pituitary axis (41), with higher levels of leptin primarily associated with appetite suppression. This may explain why women with PCOS in the present study, who usually have a tendency to gain weight and develop IR, had lower levels of leptin and presumably a greater appetite. This result is not in agreement with the results of Houjeghani et al (42) who revealed that women with PCOS had higher levels of leptin. The present study, however, was performed on a larger population. The difference in the association between leptin and PCOS in the present investigation compared with other studies may result from ethnic differences in the patients with PCOS.

In conclusion, to the best of our knowledge, the present study was the first to demonstrate that serum homocysteine levels are elevated in women with PCOS in Jordan. It was additionally revealed that compared with other metabolites previously reported to be associated with PCOS, including adiponectin, leptin and BCAAs, serum homocysteine is the most strongly associated with the PCOS phenotype. These results imply that lowering serum homocysteine may be used for the management of PCOS and/or aid in its diagnosis. This requires, however, larger studies across multiple institutions in the country.

\section{Acknowledgements}

Not applicable.

\section{Funding}

The present study was supported by the Deanship of Research at Jordan University of Science and Technology (grants nos. 214/2017 and 283/2017).

\section{Availability of data and materials}

The datasets generated and/or analyzed during the current study are available from the corresponding author on reasonable request.

\section{Authors' contributions}

All the authors participated in the design, analysis of the data and final review of the manuscript. MAA and NS conceived the study. HM, AD and MN helped with data collection. YSK and RS performed the statistical analysis. HM performed all the biochemical measurements. MAA and NS drafted the manuscript. All authors read and approved the final manuscript.

\section{Ethical approval and consent to participate}

All procedures performed in studies involving human participants were in accordance with the ethical standards of Jordan University of Science and Technology and King Abdullah University Hospital institutional review board and with the 1964 Helsinki declaration and its later amendments or comparable ethical standards. Informed consent was obtained from all individual participants included in the study.

\section{Patient consent for publication}

Written informed consent was obtained from all individual participants included in the study.

\section{Competing interests}

The authors declare that they have no competing interests.

\section{References}

1. Norman RJ, Dewailly D, Legro RS and Hickey TE: Polycystic ovary syndrome. Lancet 370: 685-697, 2007.

2. Lizneva D, Suturina L, Walker W, Brakta S, Gavrilova-Jordan L and Azziz R: Criteria, prevalence, and phenotypes of polycystic ovary syndrome. Fertil Steril 106: 6-15, 2016

3. Moran LJ, Misso ML, Wild RA and Norman RJ: Impaired glucose tolerance, type 2 diabetes and metabolic syndrome in polycystic ovary syndrome: A systematic review and meta-analysis. Hum Reprod Update 16: 347-363, 2010.

4. Diamanti-Kandarakis E and Dunaif A: Insulin resistance and the polycystic ovary syndrome revisited: An update on mechanisms and implications. Endocr Rev 33: 981-1030, 2012.

5. Badawy A and Elnashar A: Treatment options for polycystic ovary syndrome. Int J Womens Health 3: 25-35, 2011. 
6. Toulis KA, Goulis DG, Farmakiotis D, Georgopoulos NA Katsikis I, Tarlatzis BC, Papadimas I and Panidis D: Adiponectin levels in women with polycystic ovary syndrome: A systematic review and a meta-analysis. Hum Reprod Update 15: 297-307, 2009.

7. Telli MH, Yildirim M and Noyan V: Serum leptin levels in patients with polycystic ovary syndrome. Fertil Steril 77: 932-935, 2002

8. Li M, Yang M, Zhou X, Fang X, Hu W, Zhu W, Wang C, Liu D, Li S, Liu H, et al: Elevated circulating levels of irisin and the effect of metformin treatment in women with polycystic ovary syndrome. J Clin Endocrinol Metab 100: 1485-1493, 2015.

9. Lynch CJ and Adams SH: Branched-chain amino acids in metabolic signalling and insulin resistance. Nat Rev Endocrinol 10: 723-736, 2014.

10. Meigs JB, Jacques PF, Selhub J, Singer DE, Nathan DM, Rifai N, D'Agostino RB Sr and Wilson PW; Framingham Offspring Study: Fasting plasma homocysteine levels in the insulin resistance syndrome: The Framingham offspring study. Diabetes Care 24 $1403-1410,2001$

11. Buysschaert M, Dramais AS, Wallemacq PE and Hermans MP: Hyperhomocysteinemia in type 2 diabetes: Relationship to macroangiopathy, nephropathy, and insulin resistance. Diabetes Care 23: 1816-1822, 2000.

12. Selhub J: Homocysteine metabolism. Annu Rev Nutr 19: 217-246, 1999.

13. Finkelstein JD: The metabolism of homocysteine: Pathways and regulation. Eur J Pediatr 157 (Suppl 2): S40-S44, 1998.

14. Chiang EPI, Wang YC, Chen WW and Tang FY: Effects of insulin and glucose on cellular metabolic fluxes in homocysteine transsulfuration, remethylation, S-adenosylmethionine synthesis, and global deoxyribonucleic acid methylation. J Clin Endocrino Metab 94: 1017-1025, 2009.

15. Lievers KJ, Boers GH, Verhoef P, den Heijer M, Kluijtmans LA, van der Put NM, Trijbels FJ and Blom HJ: A second common variant in the methylenetetrahydrofolate reductase (MTHFR) gene and its relationship to MTHFR enzyme activity, homocysteine, and cardiovascular disease risk. J Mol Med (Berl) 79: $522-528,2001$

16. Liew SC and Gupta ED: Methylenetetrahydrofolate reductase (MTHFR) C677T polymorphism: Epidemiology, metabolism and the associated diseases. Eur J Med Genet 58: 1-10, 2015.

17. Grodnitskaya EE and Kurtser MA: Homocysteine metabolism in polycystic ovary syndrome. Gynecol Endocrinol 28: 186-189, 2012.

18. Salehpour S, Manzor-Al-Ajdad O, Samani EN and Abadi A Evaluation of homocysteine levels in patients with polycystic ovarian syndrome. Int J Fertil Steril 4: 168-171, 2011.

19. Orio F Jr, Palomba S, Di Biase S, Colao A, Tauchmanova L, Savastano S, Labella D, Russo T, Zullo F and Lombardi G: Homocysteine levels and C677T polymorphism of methylenetetrahydrofolate reductase in women with polycystic ovary syndrome. J Clin Endocrinol Metab 88: 673-679, 2003.

20. Alfaqih MA, Khader YS, Al-Dwairi AN, Alzoubi A, Al-Shboul O and Hatim A: Lower Levels of Serum Adiponectin and the T Allele of rs1501299 of the ADIPOQ Gene Are Protective against Polycystic Ovarian Syndrome in Jordan. Korean J Fam Med 39: 108-113, 2018

21. Rotterdam ESHRE/ASRM-Sponsored PCOS consensus workshop group: Revised 2003 consensus on diagnostic criteria and long-term health risks related to polycystic ovary syndrome (PCOS). Hum Reprod 19: 41-47, 2004.

22. Faul F, Erdfelder E, Lang A-G and Buchner A: G*Power 3: A flexible statistical power analysis program for the social, behavioral, and biomedical sciences. Behav Res Methods 39: 175-191, 2007.

23. Chongsuvivatwong V: Analysis of epidemiological data using $\mathrm{R}$ and Epicalc. Epidemiology Unit, Prince of Songkla University, Thailand, p328, 2008

24. Brown KS, Kluijtmans LAJ, Young IS, Murray L, McMaster D, Woodside JV, Yarnell JW, Boreham CA, McNulty H, Strain JJ, et al: The 5,10-methylenetetrahydrofolate reductase C677T polymorphism interacts with smoking to increase homocysteine. Atherosclerosis 174: 315-322, 2004.
25. Diamanti-Kandarakis E and Christakou CD: Insulin resistance in PCOS. In: Diagnosis and Management of Polycystic Ovary Syndrome. Springer, New York, NY, pp35-61, 2009.

26. Padmanabhan V and Veiga-Lopez A: Animal models of the polycystic ovary syndrome phenotype. Steroids 78: 734-740, 2013.

27. Toole JF, Malinow MR, Chambless LE, Spence JD, Pettigrew LC, Howard VJ, Sides EG, Wang CH and Stampfer M: Lowering homocysteine in patients with ischemic stroke to prevent recurrent stroke, myocardial infarction, and death: The Vitamin Intervention for Stroke Prevention (VISP) randomized controlled trial. JAMA 291: 565-575, 2004.

28. Toulis KA, Goulis DG, Mintziori G, Kintiraki E, Eukarpidis E, Mouratoglou SA, Pavlaki A, Stergianos S, Poulasouchidou M, Tzellos TG, et al: Meta-analysis of cardiovascular disease risk markers in women with polycystic ovary syndrome. Hum Reprod Update 17: 741-760, 2011

29. Moustapha A, Naso A, Nahlawi M, Gupta A, Arheart KL, Jacobsen DW, Robinson K and Dennis VW: Prospective study of hyperhomocysteinemia as an adverse cardiovascular risk factor in end-stage renal disease. Circulation 97: 138-141, 1998.

30. Wald DS, Law M and Morris JK: Homocysteine and cardiovascular disease: Evidence on causality from a meta-analysis. BMJ 325: 1202-1206, 2002

31. Brattström L and Wilcken DE: Homocysteine and cardiovascular disease: Cause or effect? Am J Clin Nutr 72: 315-323, 2000.

32. Wijeyaratne CN, Seneviratne RA, Dahanayake S, Kumarapeli V, Palipane E, Kuruppu N, Yapa C, Seneviratne RA and Balen AH: Phenotype and metabolic profile of South Asian women with polycystic ovary syndrome (PCOS): Results of a large database from a specialist Endocrine Clinic. Hum Reprod 26: 202-213, 2011.

33. Dunaif A, Sorbara L, Delson R and Green G: Ethnicity and polycystic ovary syndrome are associated with independent and additive decreases in insulin action in Caribbean-Hispanic women. Diabetes 42: 1462-1468, 1993.

34. Meng Y, Chen X, Peng Z, Liu X, Sun Y and Dai S: Association between high serum homocysteine levels and biochemical characteristics in women with polycystic ovarian syndrome: A systematic review and meta-analysis. PLoS One 11: e0157389, 2016.

35. Zhao Y and Qiao J: Ethnic differences in the phenotypic expression of polycystic ovary syndrome. Steroids 78: 755-760, 2013.

36. Gibson-Helm M, Teede H, Dunaif A and Dokras A: Delayed diagnosis and a lack of information associated with dissatisfaction in women with polycystic ovary syndrome. J Clin Endocrinol Metab 102: 604-612, 2017.

37. Cree-Green M: Worldwide dissatisfaction with the diagnostic process and initial treatment of PCOS. J Clin Endocrinol Metab 102: 375-378, 2017

38. Fulghesu AM, Porru C and Canu E: Diagnosis of Polycystic Ovarian Syndrome in Adolescence. In: Good Practice in Pediatric and Adolescent Gynecology. Springer, pp143-159, 2018.

39. Boyle JA and Teede HJ: PCOS: Refining diagnostic features in PCOS to optimize health outcomes. Nat Rev Endocrinol 12: 630-631, 2016.

40. Cammisotto PG and Bendayan M: Leptin secretion by white adipose tissue and gastric mucosa. Histol Histopathol 22: 199-210, 2007

41. Klok MD, Jakobsdottir S and Drent ML: The role of leptin and ghrelin in the regulation of food intake and body weight in humans: A review. Obes Rev 8: 21-34, 2007.

42. Houjeghani S, Pourghassem Gargari B and Farzadi L: Serum leptin and ghrelin levels in women with polycystic ovary syndrome: Correlation with anthropometric, metabolic, and endocrine parameters. Int J Fertil Steril 6: 117-126, 2012. 